\title{
Research on Characteristics of Perpetrators and casualties in Road Traffic Accidents in China-Based on the panel data analysis of national traffic accidents from 2006 to 2019
}

\author{
Lilu Sun ${ }^{1, a^{*}}$, Zhijin Zhang ${ }^{2, b}$, Mengwei Chen², , and Sen Dong ${ }^{2, \mathrm{~d}}$ \\ ${ }^{1}$ School of Management, Chongqing University of Technology, Chongqing, China \\ ${ }^{2}$ School of Economy and Finance, Chongqing University of Technology, Chongqing, China
}

\begin{abstract}
Under the background of the rapid development of Internet technology, the emergence of Internet platform accelerates the industrial agglomeration. As a chain connecting the material circulation between industries, the rise of logistics industry is inevitable. As a key link of logistics industry, transportation, along with the rise of logistics industry, has led to an increase in traffic accidents year by year. Therefore, based on the national road traffic accident data from 2006 to 2019, this paper uses SPSS 26.0 to analyse the accident incidence, casualties and direct property loss indicators of different regions and occupation, and study the characteristics of perpetrators and casualties. The results show that the traffic accidents in the whole country show a downward trend, but they are unbalanced. Workers and farmers accounted for the largest proportion, but the death and injury rate showed a downward trend. Other showed an upward trend in the past five years, especially in the delivery industry. Future road traffic safety management should take corresponding measures to reduce the accident rate from the perspective of the development of related industries.
\end{abstract}

\section{Introduction}

Road traffic accidents are the most common cause of death among accidental injuries, causing huge losses to the social economy and posing a major threat to the lives and health of residents. According to the statistics of the World Health Organization, about 1.3 million people die in traffic accidents every year in the world ${ }^{[1]}$. In addition, according to the survey report, the number of motor vehicles in developing countries accounts for $54 \%$ of the global total, but $90 \%$ of road traffic accidents occur in developing countries, indicating that there is an interdependent relationship between traffic safety and economic growth. The traffic accident mortality rate will increase first and then decrease with the economic development ${ }^{[2]}$. As the largest developing country in the world, China is now one of the countries with the highest burden of road traffic injury diseases and mortality rate in the world, and its potential loss of years of production life exceeds all other diseases and has become a major public health problem ${ }^{[3]}$. With the rapid development of economy and science and technology, China's motorization level and road modernization level have been significantly improved. Until 2019, the accumulated investment in road transportation has reached 218.5 billion yuan, and the number of civilian cars has increased from 36.9735 million in 2006 to 253.7638 billion yuan in 2019 , which is 6.86 times that of 2006. The road mileage has increased from 3.457 million kilometers in 2006 to 5.0125 million kilometers in 2019 , with a growth rate of $45 \%$. However, the rapid economic development has also brought about industrial agglomeration. In order to obtain jobs, the population has flocked to industrial agglomeration areas, which has brought about population agglomeration. Population agglomeration has led to an increase in population density, which has also brought severe road traffic safety problems. In particular, the rise of the logistics industry, in which the transportation link is closely related to the traffic, has greatly increased the traffic flow and brought a lot of pressure to the traffic accident management. Therefore, the traffic accident management has become the focus of our country's traffic field reform.

Due to the randomness, dynamics and complexity of road traffic accidents, many scholars have conducted research on the causes of road traffic accidents. Many research results show that human factors are the most important factors leading to frequent accidents. You Bingyu, Lian Fumian and others (2019) used fault tree and Bayesian network method to analyze the causes of mountainous expressway. The results show that the contribution rate of human factors to accidents reaches $54.4 \%{ }^{[4]}$. Ren Ying and Peng Hongxing (2013) analyzed macro and micro influencing factors of traffic accident death toll through fixed effects model and found that human factors account for the highest proportion of road

*Corresponding author: ${ }^{\text {"linda66@163.com, bjingbaizi@163.com, }{ }^{c} 1547750004 @ q q . c o m,{ }^{d} 15621313370 @ 163 . c o m}$ 
traffic accident casualties in all factors ${ }^{[5]}$. At present, most studies mainly analyze the causes of traffic accidents from four aspects: people, vehicles, roads and environment, or explore the correlation between the characteristics of perpetrators and casualties and the degree of traffic accidents casualties, but few studies analyze the group characteristics of national traffic accident personnel in recent years. Based on this, this paper explores the overall development trend of traffic accident personnel in recent 14 years by combing the traffic accidents in China from 2006 to 2019. The analysis of differences among regions and occupation will provide key data support for the prevention and control of road traffic accidents. While promoting the development of China's traffic management system and improving the traffic system, it also provides constructive suggestions for ensuring traffic safety from the perspective of industrial development.

\subsection{Human factors in road traffic accidents}

Among the many factors that cause traffic accidents, the human factor is the most important one. Therefore, in order to improve the traffic situation and establish a perfect traffic system, it is necessary to analyze the group characteristics of people involved in road traffic accidents. Kong Lingzheng (2013) analyzed 10 human factors affecting road traffic safety. The research results found that low driving age, drunk driving, fatigue driving, incorrect use of vehicle safety facilities and other factors are the main factors affecting road traffic safety ${ }^{[6]}$. Zhang Lixia and others (2014) believed that the driver factor was the main component of the human factor. Therefore, the impact of different driving ages on traffic accidents was mainly studied. The results showed that the driving age had a negative correlation with the number of deaths in traffic accidents ${ }^{[7]}$. Shi Xiaojie et al. (2018) found that the proportion of male drivers is 5.7 times higher than that of female drivers in major traffic accidents through comparative analysis of driver characteristics. The accident rate of drivers having 6 to 10 years driving experience is $47.06 \%$, and the accident rate of drivers aged 31 to 40 years is the highest, reaching $41.18 \%{ }^{[8]}$. Lu Renfu et al. (2020) extracted 8003 cases from the trauma database of Chongqing Emergency Medical Center. Through analysis, it was found that traffic accidents are the main cause of injury in China, among which elderly injuries are of particular concern. The number of traffic accidents for the elderly over 60 years old is inverted U-shaped, with 75 years old as the highest point ${ }^{[9]}$. Dai Long et al. (2017) used descriptive analysis to analyze the traffic accidents of children in Huli District of Xiamen. It was found that the treatment rate of traffic accidents of children showed a downward trend from 2010 to 2014. The number of injuries of boys was higher than that of girls. The high-risk age for traffic accidents of children was 5-9 years old ${ }^{[10]}$.

The spatial attribute of road system is one of the important factors affecting road traffic accidents.
Studying the spatial distribution of traffic accidents and strengthening the supervision of the areas of high frequency traffic accidents will also help to reduce the occurrence of traffic accidents. Lu Huapu et al. (2019) used density analysis and cluster analysis to identify the areas with frequent traffic accidents and high accident severity in Shenzhen. The results showed that the traffic accident severity in the suburbs was generally higher than that in the city center ${ }^{[11]}$. Fan Pengcheng et al. (2019) analyzed from the level of urban road network and adopted spatial clustering method to identify the characteristics of accident-prone areas. the results showed that traffic accidents mostly occurred on ordinary urban roads, among which ordinary road sections accounted for a relatively high proportion, reaching $75 \%{ }^{[12]}$.

Whether it is the analysis of human factors of road traffic accidents or the analysis of spatial distribution, previous studies were mostly aimed at a certain region or a certain year, but the demographic analysis of the casualties in traffic accidents in recent years across the country is relatively less. Therefore, this paper will test the differences in region and occupation of the casualties based on the traffic accident data from 2006 to 2019 across the country.

\subsection{Road traffic accident group analysis method}

For the analysis of group characteristics of road traffic accidents, the common methods are chi-square test, K-means clustering method, density analysis method, principal component analysis method, Logistic regression model, etc. Chi-square test is the most frequently used and the analysis result is more accurate. Lu Huapu et al. (2019) used density analysis method and cluster analysis method respectively to analyze the spatial distribution characteristics of urban traffic accidents. The research results show that the conclusion of density analysis method is more intuitive, and the conclusion of cluster analysis method is more accurate [11]. Wang Zhuo et al. (2018) used Chi-square test and trend test respectively to make a group analysis of some traffic casualties in Tianjin in 2014, and accurately and specifically analyzed and compared the age, occupation, geographical distribution, time distribution and cause distribution of traffic casualties ${ }^{[13]}$. As the main purpose of this study is to analyze the group characteristics of the people involved in road traffic accidents, the univariate ANOVA test can be used to test the differences between groups of each group as well as trend analysis.

\section{Materials and Methods}

\subsection{Data sources}

The selected data come from the website of the National Bureau of Statistics (http://www.stats.gov.cn/) and the Statistical Annual Report of Road Traffic Accidents of 
the People's Republic of China issued by the Ministry of Public Security of China. The selected sample range is the panel data of 31 provincial administrative regions in China from 2006 to 2019. Summarizing four data of road including the number of traffic accidents, deaths, injuries and direct property losses, and further classifying the region and occupation data of perpetrators and casualties.

\subsection{Research methods}

Firstly, the collected data are grouped according to the regional location: six groups in North China (Beijing, Tianjin, Hebei, Shanxi, Inner Mongolia), Northeast China (Liaoning, Jilin, Heilongjiang), East China (Shanghai, Jiangsu, Zhejiang, Shandong, Jiangxi, Fujian, Anhui), South China (Henan, Hunan, Hubei, Guangdong, Guangxi, Hainan), Southwest China (Chongqing, Sichuan, Guizhou, Yunnan, Tibet) and Northwest China (Shaanxi, Gansu, Ningxia, Qinghai, Xinjiang). Then, the data of perpetrators and casualties are analyzed by region and industry according to the grouping.

\subsection{Statistical analysis}

Discrete data is expressed by median, and continuous data is expressed by mean standard deviation. SPSS 26.0 software was used to test the data by ANOVA, F test was used for comparison between groups, LSD difference test was used for comparison of relative index ratio, and $\mathrm{P}<0.05$ showed significant statistical significance.

\section{Results and Discussion}

\subsection{Analysis of Traffic Accident Characteristics in Six Major Areas of China}

The whole country is divided into six regions: North China, Northeast China, East China, South China, Southwest China and Northwest China. On the whole, the number of traffic accidents, the number of injuries, the number of deaths and direct property losses in the six regions of the country all show a downward trend. The total number of traffic accidents decreased from 378,781 in 2006 to 247,646 in 2019, and an overall drop rate is $34.62 \%$, with a significant drop from 2006 to 2015. From 2016 to 2019 , there was a slight rebound, with the number of injured persons decreased from 431139 in 2006 to 275125 in 2019 , with a significant decrease rate of $36.19 \%$ and a steady decrease from 2006 to 2015. However, there was a slight fluctuation from 2016 to 2018 , and the decrease began again in 2019. The death toll decreased from 89,455 in 2006 to 62,763 in 2019 , with a decrease rate of $29.84 \%$. It decreased year by year from 2006 to 2015, increased slightly in 2016 and 2017 , and continued to decrease in 2018 and 2019. Direct property losses decreased steadily from 2006 to 2009 , with obvious fluctuations from 2010 to 2015, increased steadily from 2016 to 2018 , and decreased in 2019, with an overall decline rate of $10.65 \%$. On the whole, except for the frequent fluctuations of direct property losses from 2006 to 2019, the number of traffic accidents, injuries and deaths basically showed a significant downward trend from 2006 to 2015, with slight fluctuations from 2016 to 2019 . On the whole, the overall road traffic safety situation in China remained stable until 2019, and the four indicators of traffic accidents all showed significant decline.

Using SPSS to analyze the four indicators of traffic accidents, deaths, injuries and direct property losses, this paper compares the data of six major areas from 2006 to 2019 in the past 14 years and adopts difference analysis to test whether there are differences and their extent among different areas.

This paper uses the one-way analysis of variance. The prerequisite of this method is the homogeneity of the variance of the parameters. Therefore, the homogeneity of the variance of the parameters is tested. According to the results of variance homogeneity test, the result of $\mathrm{F}$ test is $\mathrm{P}<0.05$ in four indexes: the number of traffic accidents, the number of deaths, the number of injured people and the direct property loss, so the variance homogeneity test among the six major regions is homogeneous, so single factor ANOVA test can be used. According to the results of ANOVA test, $\mathrm{P}<0.05$, indicating that there are significant differences among the groups of the four indicators of traffic accidents, injuries, deaths and direct property losses, and two-to-two comparison is required. Therefore, the LSD method is adopted to make multiple comparisons among the six major areas, and the comparison results are as follows:

Table 1. Multiple comparison of the number of traffic accidents in six major areas of China

\begin{tabular}{|c|c|c|c|c|c|c|}
\hline $\mathrm{R}$ & $\mathrm{NC}$ & $\mathrm{NE}$ & $\mathrm{EC}$ & $\mathrm{CN}$ & SW & NW \\
\hline \multicolumn{7}{|l|}{$\mathrm{NC}$} \\
\hline $\mathrm{NE}$ & $\begin{array}{c}0.028 \\
*\end{array}$ & & & & & \\
\hline $\mathrm{EC}$ & $\begin{array}{c}0.000 \\
* *\end{array}$ & $\begin{array}{c}0.000 \\
* *\end{array}$ & & & & \\
\hline CS & $\begin{array}{l}0.000 \\
* *\end{array}$ & $\begin{array}{l}0.000 \\
* *\end{array}$ & $\begin{array}{c}0.002 \\
* *\end{array}$ & & & \\
\hline SW & 0.391 & $\begin{array}{c}0.003 \\
* *\end{array}$ & $\begin{array}{c}0.000 \\
* *\end{array}$ & $\begin{array}{c}0.000 * \\
*\end{array}$ & & \\
\hline NW & 0.126 & 0.491 & $\begin{array}{c}0.000 \\
* *\end{array}$ & $\begin{array}{c}0.000 * \\
*\end{array}$ & $\begin{array}{c}0.018 \\
*\end{array}$ & \\
\hline
\end{tabular}

(* means significant difference at 0.05 significance level, $* *$ means significant difference at 0.01 significance level)

( $\mathrm{NC}$ represents North China, NE represents Northeast China, EC represents East China, CS represents Central and South China, SW represents Southwest China, and NW represents Northwest China) 


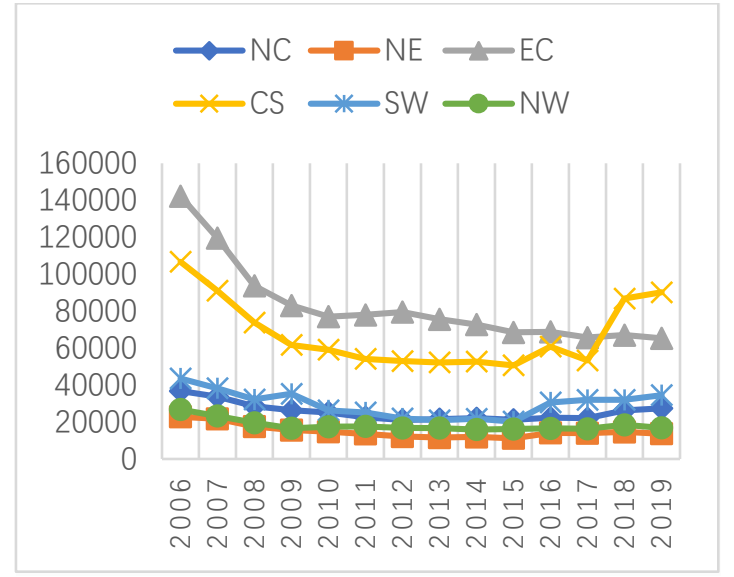

Fig. 1. Number of traffic accidents in six regions of China from 2006 to 2019

As can be seen from Table1, in terms of traffic accidents, the difference between East China and other regions is the most significant, satisfying the $99 \%$ confidence interval. There is a significant difference between Central South China and the other five regions at the significance level of 0.05 . Besides North China, there is also a significant difference between Southwest China and the other four regions at the significance level of 0.05. In addition, except for Northwest China, there is a significant difference between Northeast China and other regions at the significance level of 0.05 , while there is no significant difference between North China and Northwest China. At the same time, as can be seen from Figure 1, the number of traffic accidents in the six major regions of the country, on the whole, showed a decreasing trend. From 2006 to 2017, the number of traffic accidents in East China was significantly higher than that in other regions. The decrease rate from 2006 to 2008 was relatively fast, with a decrease rate of $34.13 \%$. From 2017 to 2018, the number of traffic accidents in Central South China increased sharply, with a growth rate of $68.89 \%$. At the same time, the number of traffic accidents in Central South China is second only to that in East China. The number of traffic accidents in the other four districts decreased by a relatively small margin and fluctuated slightly during the decline. The number of traffic accidents in the southwest region increased slightly in 2009 and began to fluctuate from 2015. North China experienced a small fluctuation from 2013 to 2016 , with a small increase of $24.32 \%$ after 2017 , while northeast China experienced a small fluctuation from 2014. East China experienced a slight increase in 2011 and 2012, with a year-on-year decrease from 2013 to 2015 and a small fluctuation from 2016 to 2019 .

Table 2. Multiple Comparison of Injuries in Six Regions of the Country

\begin{tabular}{ccccccc}
\hline $\mathrm{R}$ & $\mathrm{NC}$ & $\mathrm{NE}$ & $\mathrm{EC}$ & $\mathrm{CN}$ & $\mathrm{SW}$ & $\mathrm{NW}$ \\
\hline $\mathrm{NC}$ & & & & & & \\
$\mathrm{NE}$ & 0.050 & & & & &
\end{tabular}

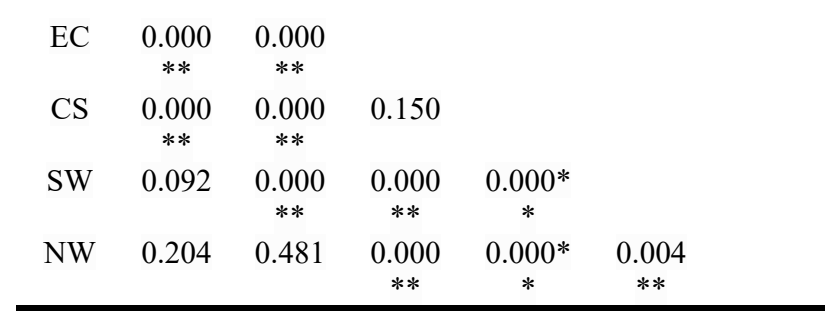

In terms of the number of injured people, except for East China and South China, there is no significant difference between North China and other regions, nor is there any significant difference between South China and East China, and between Northeast and Northwest regions. In addition, there are significant differences at the significance level of 0.01 in other regions. The overall trend of the number of traffic accidents injuries in the six major regions of the country is declining, among which the number of traffic accidents injuries in East China increased slightly in 2012, 2016 and 2018. Before 2017, the number of traffic accidents injuries in the six major regions ranked first. After 2017, the number of injuries in Central southern China increased sharply, surpassing that in eastern China, ranking first with a growth rate of $62.89 \%$. From 2006 to 2019, the southwest region fluctuates more, reaching its highest point in 2018, with a month-on-month growth rate of $42.82 \%$. The other three regions are relatively stable. Among them, the number of injuries in North China is more than that in the other two regions. After 2013, it began to fluctuate. The growth rates in 2018 and 2019 are larger than those in previous years. There are many small fluctuations in Northeast China and Northwest China.

Table 3. Multiple Comparison of Deaths in Six Regions of the Country

\begin{tabular}{ccccccc}
\hline $\mathrm{R}$ & $\mathrm{NC}$ & $\mathrm{NE}$ & $\mathrm{EC}$ & $\mathrm{CN}$ & $\mathrm{SW}$ & $\mathrm{NW}$ \\
\hline $\mathrm{NC}$ & & & & & & \\
$\mathrm{NE}$ & 0.000 & & & & & \\
& $* *$ & & & & & \\
$\mathrm{EC}$ & 0.000 & 0.000 & & & & \\
& $* *$ & $* *$ & & & & \\
$\mathrm{CS}$ & 0.000 & 0.000 & 0.000 & & \\
& $* *$ & $* *$ & $* *$ & & & \\
$\mathrm{SW}$ & 0.963 & 0.000 & 0.000 & $0.000^{*}$ & \\
& & $* *$ & $* *$ & $*$ & \\
$\mathrm{NW}$ & 0.024 & 0.142 & 0.000 & $0.000^{*}$ & 0.027 & \\
& $*$ & & $* *$ & $*$ & $*$ \\
\hline
\end{tabular}

In terms of death toll, there is no significant difference between North China and Southwest China, nor is there any significant difference between Northeast China and Southwest China, and there are significant differences in other regions at the significance level of 0.05 . Among the six major regions in the country, the death toll in East China and Central South China is significantly higher than that in other regions, with East China taking the first place, followed by Central South China. From 2006 to 2016, the death toll in East China is 
significantly higher than that in Central South China. After 2017, the two areas tend to be consistent. In the other four regions, before 2013, the death toll in North China was higher than that in other regions, and the decrease was significant in 2018. After 2013, the death toll in Southwest China exceeded that in North China, with a significant increase. In addition, the trend in Northeast and Northwest China was gentle with small fluctuations.

Table 4. Multiple Comparisons of Direct Property Losses in Six Regions of the Country

\begin{tabular}{ccccccc}
\hline $\mathrm{R}$ & $\mathrm{NC}$ & $\mathrm{NE}$ & $\mathrm{EC}$ & $\mathrm{CS}$ & $\mathrm{SW}$ & $\mathrm{NW}$ \\
\hline $\mathrm{NC}$ & & & & & & \\
$\mathrm{NE}$ & 0.000 & & & & & \\
& $* *$ & & & & & \\
$\mathrm{EC}$ & 0.000 & 0.000 & & & & \\
& $* *$ & $* *$ & & & & \\
$\mathrm{CS}$ & 0.000 & 0.000 & 0.001 & & & \\
& $* *$ & $* *$ & $* *$ & & & \\
$\mathrm{SW}$ & 0.575 & 0.002 & 0.000 & $0.000^{*}$ & & \\
& & $* *$ & $* *$ & $*$ & & \\
$\mathrm{NW}$ & 0.000 & 0.274 & 0.000 & $0.000^{*}$ & 0.000 & \\
& $* *$ & & $* *$ & $*$ & $* *$ & \\
\hline
\end{tabular}

In terms of direct property losses, East China, like Central and South China, has the most significant difference with other regions, all below the significance level of 0.01. Except for Southwest China, North China has significant difference with other regions at the significance level of 0.01, while Northwest China and Northeast China have no significant difference. In addition, there are significant differences among other regions. Among the six major regions in the country, North China, Central South and Southwest regions as a whole tend to increase, while the rest regions as a whole tend to decrease. Among them, East China region has the largest direct property loss, which decreased significantly from 2006 to 2010, with a slight increase from 2010 to 2012, a slight decrease from 2012 to 2016 , and a slight fluctuation after 2016. The central and southern regions began to increase after 2009, with a slight decrease from 2012 to 2013, and a sharp increase after 2015 , with a growth rate of $75.82 \%$, with a decrease in 2019. The other four regions experienced more fluctuations, among which the southwest region increased significantly after 2015, surpassing the north China region, and the northwest region had the lowest direct property losses from 2006 to 2019.

In addition, through statistics, it is found that the average mortality rates in Central South, Northwest China and East China are 25.10\%, 24.34\% and 23.47\%, respectively, which are the top three in China. The reason is that East China and Central South China are densely populated areas, with the resident population ranking first and second in China respectively, with developed economy, industrial agglomeration, large vehicle ownership and dense population. Although the transportation infrastructure is perfect, the management pressure of traffic accidents is still very great. According to relevant studies, there is a very high correlation between the number of traffic accident deaths and the population, with a correlation coefficient of $0.945^{[14]}$. Therefore, the average death rate of traffic accidents is in the forefront. As for Northwest China, its resident population is not dense, and the number of traffic accidents is less than other regions, but the average mortality rate is second. Reason lies in the poor economic development and imperfect medical industry in Northwest China, which is not only manifested in the lack of medical infrastructure and medical equipment, but also in the lack of medical level, so the average mortality rate in Northwest China is higher.

\subsection{Occupational Analysis of Traffic Accident-Related Personnel in China}

In this paper, the occupations of the involved personnel are divided into 6 groups, and the variance test of each group is homogeneous $(\mathrm{P}<0.05)$. Therefore, LSD test is carried out on the 6 groups. Tables 9 and 10 shows that there are significant differences in the three indicators of traffic accidents, deaths and injuries between workers and the other 5 groups, peasants and the other 5 groups, which are in line with the $99 \%$ confidence interval. there is no significant difference in direct property losses between workers and other. There are significant differences in the four indicators between minor students and workers and farmers, minor students and others. Except for ordinary staff and minor students, there are significant differences between self-employed and other groups at the significance level of 0.01 . From 2006 to 2016, the number of traffic accidents among workers and farmers was significantly higher than that of other groups. In line with this, before 2019, the number of casualties among workers and farmers was much higher than that of other groups, with the overall trend declining. From 2006 to 2012 , the decline rate was $38.48 \%$, and it leveled off after 2013. The direct property index also fluctuated and declined after 2012. The number of traffic accidents in other was second only to that of workers and farmers. After 2015, the number of casualties rose sharply, leading to a significant increase in the number of casualties. After 2018, the number of casualties surpassed that of workers and farmers, with an increase rate of $44.89 \%$. This led to a sharp increase in direct property losses, with the highest point being four times that of other industries. The overall trend of other four industries in the four indicators was downward, with slight fluctuations during the period. However, the number of injuries among minor students increased in the past two years.

It is worth noting that the four indicators of other industries have suddenly increased since 2015, surpassing the other five industries, especially the take-away industry, which was separately reported in the 2019 traffic annual report, indicating that the traffic accidents in this industry have been noticed by the transportation department. As an important part of the logistics industry, the data of the take-away industry can 
reflect the traffic accidents in the logistics industry, especially transportation is an important part of the logistics industry. Transportation is closely related to traffic, so it is necessary to improve the management and operation of logistics industry in order to reduce the loss of personnel and property by analyzing traffic accident data. This paper argues that the sudden increase of the four indicators of traffic accidents may be related to the national attention to the "internet plus" strategy and digital economy in recent years. The maturity of Internet technology leads to the emergence of platform economy. As a business model innovation, platform economy has realized the deep integration of Internet technology and real economy, changed the traditional industrial chain supply and marketing organization mode, and promoted the upgrading of industrial structure ${ }^{[15]}$. It is inevitable that both the take-out industry and the logistics industry will rise under the platform economy, but the management of traffic safety has not responded to the rapid development of the logistics industry in time, which leads to a sharp increase in traffic accidents in this industry in recent years. Therefore, other industries will have an abnormal increase in the four indicators.

Table 5. Multi-industry Comparison of Traffic Accidents in China

\begin{tabular}{|c|c|c|c|c|c|c|}
\hline$\overline{\mathrm{R}}$ & $\overline{C S}$ & $\overline{\mathrm{OE}}$ & $\overline{W F}$ & $\overline{\mathrm{SE}}$ & $\overline{U S}$ & Other \\
\hline \multicolumn{7}{|l|}{$\mathrm{CS}$} \\
\hline $\mathrm{OE}$ & 0.042 & & & & & \\
\hline WF & $\begin{array}{c}0.000 \\
* *\end{array}$ & $\begin{array}{c}0.000 \\
* *\end{array}$ & & & & \\
\hline $\mathrm{SE}$ & $\begin{array}{c}0.000 \\
* *\end{array}$ & 0.013 & $\begin{array}{c}0.000 \\
* *\end{array}$ & & & \\
\hline US & 0.839 & 0.026 & $\begin{array}{c}0.000 \\
* *\end{array}$ & $\begin{array}{c}0.000 * \\
*\end{array}$ & & \\
\hline Othe & 0.000 & 0.000 & 0.000 & $0.000 *$ & 0.000 & \\
\hline $\mathrm{r}$ & $* *$ & $* *$ & $* *$ & $*$ & $* *$ & \\
\hline
\end{tabular}

\section{Suggestions}

In recent years, China's traffic accident management system has become increasingly perfect and has achieved remarkable results in the implementation process. However, traffic accidents have rebounded slightly in the past two years. Therefore, it is urgent to further improve the traffic management system. The government and academic circles emphasize the important role of human factors in traffic accident control [16], Li Xiaojuan (2006) ${ }^{[17]}$ and Jia Wang (2017) ${ }^{[18]}$ analyze the relationship between the number of traffic accidents, casualties and traffic accident losses, and propose a traffic accident loss prediction method based on BP neural network. Liu Yin (2008) ${ }^{[19]}$ based on the accident cause theory, analyzes the causes of traffic accident losses, emphasizes the equal position of people and things in the accident cause, and puts forward the corresponding control strategies. Based on the analysis of the characteristics of traffic perpetrators from 2006 to 2019 in recent fourteen years, this paper puts forward the following suggestions.

(A) Establish an intelligent transportation system.

Traffic congestion is also the cause of traffic accidents. Therefore, from the national level, we should speed up the application of the new generation of Internet technology to promote the development of "Internet plus Traffic", form an intelligent transportation system, establish an intelligent transportation system with high coverage and timely traffic real-time monitoring, feedback and control, and perceive, interconnect, analyze, predict and control traffic conditions in a larger scope to help upgrade China's overall traffic management system and technical process. At the same time, Intelligent transportation system can also be used to analyze accident causes, evolution rules, management and control strategies, and design active safety technologies and management methods, so as to realize the integration of traffic safety operation prevention and control from the perspective of man-vehicle-road coordination. The intelligent transportation system can also add the function of recording vehicle owner information. After a traffic accident, vehicle owner information can be checked in time through license plates, and family members can be contacted and sent to hospitals for treatment, thus reducing the time for hospitals to inquire patient information. Intelligent transportation system can not only be used for traffic accident prevention and rescue, but also improve traffic efficiency and reduce vehicle energy consumption, which makes transportation safer and greener and promotes the sustainable development of industry.

(B) Promote the development of smart medical industry in underdeveloped areas.

The backwardness of medical and health industry is also the main factor leading to the increase of traffic accident casualty rate. Taking Northwest China as an example, the number of traffic accidents is the last, but the average mortality rate is the second, which is closely related to the backwardness of medical industry. Therefore, it is very important for economically backward areas to promote the development of smart medical industry, and the key to promote its development lies in using the "internet plus application platform" to realize remote diagnosis and remote medical services. Because of the limited medical level, economically backward areas cannot treat seriously injured patients caused by traffic accidents, but telemedicine services can provide help and guidance for treating hospitals. Therefore, although China has formed five smart medical industry clusters in Beijing, Guangdong, Shanghai, Jiangsu and Zhejiang, its number and coverage need to be further expanded to cover all cities in China. On the other hand, most of the smart medical equipment exported by China at present is low-end equipment. Therefore, it is effective and sustainable to speed up the research and development of high-end smart medical equipment and reduce the 
dependence on imported high-end smart medical equipment for economically underdeveloped remote areas. It improves the medical level in economically underdeveloped areas, reduce the death rate of traffic accidents, and reduce the medical cost.

(C) Establishing intelligent logistics platform.

In view of the sudden increase of the four indicators of traffic accidents in the take-away industry in recent years, it can be reflected that the traffic accidents in the logistics industry are becoming more and more serious. Therefore, for the transportation link, it is necessary to build a special logistics transportation platform, use artificial intelligence to provide real-time monitoring of vehicles, introduce real-time road traffic conditions, plan the optimal driving route, save transportation costs, reduce oil consumption, and remind drivers to avoid traffic jams and drive carefully in dangerous sections, which is conducive to reducing the occurrence of traffic accidents in the logistics industry.

(D) According to local conditions to establish a regional traffic law and management system.

Strengthening law enforcement is the government's most direct and effective means of traffic management ${ }^{[20-21]}$. As mentioned above, East China and Central and South China are traffic accident-prone areas, which are significantly different from other areas. There are many serious traffic accidents, and the situation is more complicated. Therefore, it is necessary to focus on classifying the causes of the accidents and conduct full-angle detection through big data to increase law enforcement efforts and improve the efficiency of the rescue process. The number of accidents in southwest China has increased significantly in recent years. The main reason is that the terrain in southwest China is mostly rugged. Therefore, road construction should be adapted to the terrain. The economic development, road construction and people's living habits in different districts are different from each other due to the imbalance of traffic safety management. Therefore, this study suggests that an effective traffic management system should be established according to the terrain features and types of frequent accidents in the region, with emphasis on improving traffic regulations, construction of traffic legal system and enforcement of laws. In addition, in order to reduce the casualty rate of traffic accidents, the government can also improve the compulsory traffic insurance system and issue a road traffic accident social assistance fund policy to provide economic assistance to those involved in family poverty.

(E) Establish a re-education mechanism for traffic safety.

People are the only subjective and conscious subject in traffic accidents and the key to traffic safety management ${ }^{[22-23]}$. In view of the problem that traffic accidents of workers and farmers are much higher than those of other industries, traffic safety education should be carried out first. Secondly, for the floating population in this group, because they do not have a strong sense of belonging to the working city and do not pay enough attention to the road safety of urban construction, they should be helped to understand the local traffic rules and enhance their awareness of traffic safety. The local government should also formulate relevant systems, such as medical and social security systems, to enhance the sense of belonging of the floating population.

\section{5 conclusion}

In this study, the characteristics of traffic accidents in China are deeply analyzed from the characteristics of human-caused perpetrators and casualties of traffic accidents, and the analysis provides the basis for traffic management from two aspects: region and occupation. and puts forward some suggestions for reducing traffic accidents and casualties from the perspective of promoting the development and improvement of medical and logistics industries. However, this paper has not conducted in-depth research and explanation on casualties and specific causes of accidents, which is the direction of our other research, that is, to subdivide the influencing factors of traffic accidents, and to verify and supplement the existing conclusions.

\section{Acknowledgments}

Fund projects: Chongqing Education Science Planning Project(2019-GX-127), Chongqing Social Science Association Project(2019YBGL067), Chongqing Education Commission Humanities and Social Sciences Research Project (19SKGH126), Chongqing Postgraduate Education Reform Research Project (yjg183113).

\section{References}

1. Zhang Hengqiu, Zhou Yong, Zeng Xiang, Zeng Chao, Chen Zeling, Zhao Yangyang, Liang Xiaodong. Retrospective analysis of road traffic injury death surveillance data in Zhuhai from 2006 to 2017 [J]. south china journal of preventive medicine,2020,46(03):287-289+293.

2. Smeed R J. The usefulness of formulae in traffic engineering and road safety [J]. Accident Analysis \& Prevention, 1972,4(4):303-312.

3. Li Yanhong, Bai Jie, Zhou Liqing, Wang Lihua, Zhou Deding, Su Huijia, Zhang Hongwei. Analysis of epidemic characteristics and related factors of traffic injuries among Shanghai residents [J]. Chinese Journal of Disease Control, 2017,21(01):65-68.

4. You.Bingyu, Lian Fumian, Meng Xianghai. Analysis of Causes of Highway Accidents in Mountain Area Based on Fault Tree Bayesian Network. Traffic Information and Safety,2019, 37(04):44-51.

5. Ren Ying, Peng Hongxing. Empirical Analysis on Influencing Factors of Traffic Accident Casualties in China [J]. Forecast,2013,32 (3): 1-7. 
6. Kong Lingzheng. Analysis of Human Factors in the Causes of Traffic Accidents [J]. China Safety Science Journal,2013,23(01):28-34.

7. Zhang Lixia, Liu Tao, Pan Fuquan, Guo Tao, Liu Ruichang. Analysis on the Influence of Driver Factors on Road Traffic Accident Indicators [J]. China Safety Science Journal, 2014,24(05):79-84.

8. Shi Xiaojie, Gao Xiannian, Jia Wang, He Jie. Research on Serious and Extreme Traffic Accidents Based on Driver Characteristics [J]. Heilongjiang Traffic Science and Technology, 2018,41(02):171-173.

9. Lu Renfu, Qiu Jun, Kong Lingwen, Hu Ping, Du Dingyuan. Epidemiological analysis of 8003 hospitalized patients with trauma [J]. journal of traumatic surgery, 2020,22(06):442-446.

10. Dai Long, Wu Xiaoqing, Zhang Jinhua, Kang Jingying. Epidemiological characteristics of road traffic injuries among children in Huli District of Xiamen [J]. Straits Journal of Preventive Medicine, 2017,23(03):23-25.

11. Lu Huapu, Luo Shengxi, Li Rumin. Research on spatial distribution characteristics of road traffic accidents in Shenzhen based on GIS analysis [J]. china journal of highway and transport, 2019,32(08):156-164.

12. Fan Pengcheng, Dong Xianyuan. Analysis and application of common characteristics of accident-prone areas of urban road network based on spatial clustering $[\mathrm{J}]$. Transportation Science and Technology, 2019, (04):85-89.

13. Wang Zhuo, Wang Dezheng, Zhang Ying, Shen Chengfeng, Zhang Hui, Song Degui, Xu Zhongliang, Jiang Guohong. Epidemiological characteristics of road traffic injury in Tianjin [J]. Chinese Journal of Disease Control, 2018,22(03):249-2

14. Huang Jie, Ye Houyuan, Yun Jun. Research on the law of traffic accidents in China based on macro econometric panel data model [J]. Safety and Environmental Engineering, 2017, 24(004):105-111.

15. Melo W A, Alarcão A $\mathrm{C}$, de Oliveira a $\mathrm{P}$, et al. Age-Related Risk Factors with Non-Fatal Traffic Accidents in Urban Areas in Maringá, Paraná, Brazil[J]. Journal of Crash Prevention \& Injury Control, 2017, 18(2):157-163.

16. Li Xiaojuan, Ding Yi, Yue Xiaoquan, Huan Xiaoting. Prediction of road traffic accident losses based on BP neural network [J]. Forest Engineering, 2006, (05):57-59.

17. Qu Xiaoxi. Research on the high-quality development of e-commerce industry from the perspective of platform economy [J/OL]. Enterprise Economics, 2021(05):63-70

18. Wang J, Yang Y, Liu S. Study of traffic characteristics based on internet web real-time traffic conditions by image identification technology[J]. Imaging Science Journal the, 2017(18):1-8.

19. Liu Yin, Lu Jianxin. Cause analysis and control strategies for road traffic accident losses [J]. Road traffic and safety, 2008, (04):14-17.

20. Apasnore P, Ismail K, Kassim A. Bicycle-vehicle interactions at mid-sections of mixed traffic streets: Examining passing distance and bicycle comfort perception[J]. Accid Anal Prev, 2017, 106:141-148.

21. Zhong-Wen L I, Wang Y G, Huang S J, et al. Effects of different interference values on fatigue properties of aluminum alloy specimens with fastener holes[J]. Light Alloy Fabrication Technology, 2017.

22. Aslani M, Mesgari M S, Wiering M. Adaptive traffic signal control with actor-critic methods in a real-world traffic network with different traffic disruption events [J]. Transportation Research Part C Emerging Technologies, 2017, 85:732-752.

23. López G, Oña $J$ D, Garach L, et al. Influence of deficiencies in traffic control devices in crashes on two-lane rural roads[J]. Accident Analysis \& Prevention, 2016, 96:130-139. 